\title{
The graph distance game and some graph operations *
}

\author{
J. Cáceres ${ }^{1}, \underline{\text { C. Hernando }}^{2}$, M. Mora ${ }^{2}$, I. M. Pelayo ${ }^{2}$, and M. L. Puertas ${ }^{1}$ \\ 1 Universidad de Almería, Almería, Spain \{jcaceres, mpuertas\}@ual.es \\ 2 Universitat Politècnica de Catalunya, Barcelona, Spain \\ \{carmen.hernando, merce.mora, ignacio.m.pelayo\}@upc.edu
}

\begin{abstract}
In the graph distance game, two players alternate in constructing a maximal path. The objective function is the distance between the two endpoints of the path, which one player tries to maximize and the other tries to minimize. In this paper we examine the distance game for various graph operations: the join, the corona and the lexicographic product of graphs. We provide general bounds and exact results for special graphs.
\end{abstract}

Key words: Distance game, graph operations.

\section{Introduction}

Combinatorial games have been widely studied and are constantly developed. Many such games are modifications of some previous ones [17], other are entirely new. Combinatorial games remain an active field of new and interesting research. For an extensive bibliography on combinatorial games and related topics see [12].

Notice that, as shown in [14], in many combinatorial games, the winner is determined by who moves last, as studied for example in [3]. In others, the players compete to construct a desired goal, by taking one element at a time from the universe, as studied for example in [2]. Another class of games consists of those where the players compete to maximize or minimize some quantity, such as the game chromatic number introduced in [11], the competition chromatic number introduced in [21], graph competition independence introduced in [18] or the domination game introduced in [4]. We consider here a game that falls in the latter category. (See [19] for more on such competitive games.)

Some of this games remained more or less unnoticed for many years, as for example, the game chromatic number. However, in the last several years

\footnotetext{
* Research partially supported by projects MTM2012-30951, Gen. Cat. DGR 2009SGR1040, Ge. Cat. DGR2014SGR46, ESF EUROCORES programme EUROGIGAComPoSe IP04-MICINN, MTM2011-28800-C02-01, Gen. Cat. DGR 2009SGR1387
} 
various authors focus their attention in this topics. Many of this combinatorial games are specially studied in some families of graphs, such as trees, forests, outerplanar graphs, cactuses or wheels $[5,6,15,20,16]$. Others authors infer interrelationship between this games and some operations in graphs $[9,1,22,10]$.

In [14], the graph distance game is introduced as follows. Given a graph $G$, two players alternate in constructing a path. The first player picks a vertex, the second player picks a neighbor of the first vertex, the first player picks a neighbor of the second vertex that has not yet been picked, and so on. This is continued until the path cannot be extended. One player tries to maximize the final distance from the start, and the other player tries to minimize this distance. That is, the value at the end of the game is the distance between the start and the finish, regardless of the path taken. We call this the distance game.

\section{Basics and known results of graph distance game}

In [14], the authors explore the distance game for various graphs and provide general bounds and exact results for simple graphs. They also show that the parameter can be calculated in a tree in linear time. Further, the values for small grids are determined.

There are two versions, depending on which of the Minimizer or Maximizer moves first. We let $S_{m}(G)$ denote the value of the game on graph $G$ when the minimizer chooses the first vertex, and $S_{M}(G)$ the value when the maximizer chooses the first vertex. We call the first vertex of the path the source. Clearly, if the graph is vertex transitive, all sources are equivalent. If it does not matter who goes first, then we drop the subscript and write $S(G)$.

For a trivial example, the value of this parameters is always 1 in the complete graph. Table 1 shows the values of these parameters for paths, cycles, complete bipartite graphs and wheels.

Table 1: Distance game parameters of some basic graphs.

\begin{tabular}{lllllll}
\hline$G$ & $P_{n}$ & $C_{n}$ & \multicolumn{1}{l}{$K_{n}$} & \multicolumn{1}{l}{$K_{p, q}$} & $W_{n}$ \\
\hline$S_{m}(G)$ & $\left\lfloor\frac{n}{2}\right\rfloor$ & 1 & 1 & 1 & $($ if $p=q)$ & 1 \\
$S_{M}(G)$ & $n-1$ & 1 & 1 & $1,2($ if $p \neq q)$ & 1 \\
\hline
\end{tabular}

The radius and the diameter of the graph give bounds of these parameters.

Proposition 1. Let $G$ be a graph of order $n$, radius $\operatorname{rad}(G)=r$ and diameter $\operatorname{diam}(G)=d$. Then, $1 \leq S_{m}(G) \leq r$ and $1 \leq S_{M}(G) \leq d$. 
In Figure 1, we show the distance game pairs $(x, y)$ of alls vertices of $P_{6}$. In these terms, $S_{m}$ is the minimum of the $x$-numbers and $S_{M}$ is the maximum of the $y$-numbers. In this example, the Maximizer choose as first vertex one of the leaves and Minimizer select one central vertex as source.

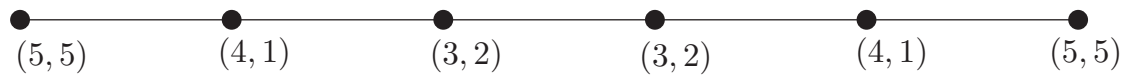

Fig. 1: Distance game pairs of all vertices of $P_{6}$. Notice that $S_{m}\left(P_{6}\right)=3$ and $S_{M}\left(P_{6}\right)=5$.

In other graphs, the same vertex could be a good source for the two players, the Maximizer and the Minimizer.

\section{Operations}

In this paper we examine the distance game for various graph operations. We follow the notation of [7].

In [14], the authors explore the distance game for small grids. Specifically, the following results were obtained.

Theorem 1. i) $S_{M}\left(P_{2} \square P_{m}\right)=1$ and $S_{m}\left(P_{2} \square P_{m}\right)=2\lfloor m / 4\rfloor+1$,

$$
\text { ii) } \begin{aligned}
S_{M}\left(P_{3} \square P_{m}\right) & =\left\{\begin{array}{l}
m+1, \text { for odd } m, \\
1, \quad \text { for even } m .
\end{array}\right. \\
S_{m}\left(P_{3} \square P_{m}\right) & =\left\{\begin{array}{l}
2, \text { for odd } m \geq 3, \\
1, \text { for even } m .
\end{array}\right.
\end{aligned}
$$
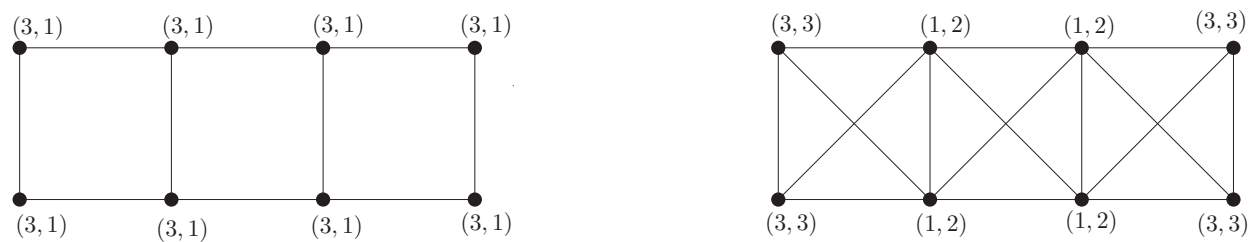

Fig. 2: Distance game pairs of vertices of the cartesian and the strong product of $P_{2}$ and $P_{4}$.

We focus our attention in the study of the distance game for join, corona and lexicographic product of graphs. 


\subsection{Join}

The join $G=G_{1} \vee G_{2}$ is the graph such that $V(G)=V\left(G_{1}\right) \cup V\left(G_{2}\right)$ and $E(G)=E\left(G_{1}\right) \cup E\left(G_{2}\right) \cup\left\{u v: u \in V\left(G_{1}\right), v \in V\left(G_{2}\right)\right\}$.

Remark 1. Notice that $1 \leq S_{m}(G \vee H) \leq 2$ and $1 \leq S_{M}(G \vee H) \leq 2$, since $1 \leq \operatorname{diam}(G \vee H) \leq 2$.

Theorem 2. Let $G, H$ be graphs. Then, $S_{m}(G \vee H)=1$.

Remark 2. Notice that $S_{M}\left(P_{2} \vee P_{3}\right)=1$ and $S_{M}\left(P_{2} \vee P_{4}\right)=2$.

\subsection{Corona product}

Let $G$ and $H$ be two graphs and let $n$ be the order of $G$. The corona product of graphs $G$ and $H$ is the graph $G \odot H$ obtained by taking one copy of $G$ and $n$ copies of $H$, and then joining by an edge the $i^{\text {th }}$ vertex of $G$ to every vertex in the $i^{\text {th }}$ copy of $H$. The corona product is neither associative nor commutative.

Theorem 3. Let $G, H$ be graphs. Then,

$$
1 \leq S_{m}(G \odot H) \leq 2 \text { and } 1 \leq S_{M}(G \odot H) \leq 3 .
$$

Remark 3. For both parameters all values are possible:

- If $H \cong\left(K_{2}+K_{2}\right) \vee K_{1}$, then $S_{m}(G \odot H)=1$.

- If $H \cong P_{3}$ (or $H \cong K_{q}$ ) and $G \nsucceq K_{1}$, then $S_{m}(G \odot H)=2$.

And

- If $H \cong K_{p}$ and $G \cong K_{1}$, then $S_{M}(G \odot H)=1$.

- If $H \cong P_{3} \odot K_{1}$, then $S_{M}(G \odot H)=2$.

- If $H \cong K_{q}$ and $G \neq K_{1}$, then $S_{M}(G \odot H)=3$.

Remark 4. Notice that

- If $H \cong P_{n}$ and $n \geq 5$, then $S_{M}(G \odot H) \leq 3<n-1=S_{M}(H)$.

- If $H \cong K_{r}$ and $G \nsubseteq K_{1}$, then $S_{M}(G \odot H)=3>1=S_{M}(H)$.

- If $H \cong P_{3} \odot K_{1}$ and $G \cong K_{r}$, then $S_{M}(G \odot H)=2>1=S_{M}(G)$.

- If $G \cong P_{n}$ with $n \geq 5$ and $H \cong K_{r}$, then $S_{M}(G \odot H)=3<n-1=S_{M}(G)$.

\subsection{Lexicographic product}

The lexicographic product of graphs $G$ and $H$ is the graph $G \circ H$ on vertex set $V(G) \times V(H)$ in which vertices $\left(g_{1}, h_{2}\right)$ and $\left(g_{2}, h_{2}\right)$ are adjacent if and only if either $g_{1} g_{2} \in E(G)$ or $g_{1}=g_{2}$ and $h_{1} h_{2} \in E(H)$. This graph operation is also known as the graph composition and denoted by $G[H]$. The graph $G \circ H$ is called nontrivial if both factors are graphs on at least two vertices. Next, we show a basic list of properties of this graph operation, whose proofs are direct consequences of the definition. 
Proposition 2. Let $G, H$ be graphs.

1. The graph $G \circ H$ is connected if and only if $G$ is connected.

2. The lexicographic product is associative but not commutative.

3. If $G$ is connected, then

- $d_{G \circ H}\left((g, h),\left(g^{\prime}, h^{\prime}\right)\right)=d_{G}\left(g, g^{\prime}\right)$ if $g \neq g^{\prime}$,

- $d_{G \circ H}\left((g, h),\left(g, h^{\prime}\right)\right)=2$ if $h h^{\prime} \in E(H)$,

- $d_{G \circ H}\left((g, h),\left(g, h^{\prime}\right)\right)=1$ if $h h^{\prime} \in E(H)$.

We have obtained the following result.

Theorem 4. Let $G, H$ be graphs. Then,

$$
1 \leq S_{m}(G \circ H) \leq S_{m}(G) \text { and } S_{M}(G) \leq S_{M}(G \circ H) .
$$

Conjecture 1. Let $G$ be a graph. Then,

$$
1 \leq S_{m}\left(G \circ K_{q}\right)=S_{m}(G) \text { and } S_{M}(G)=S_{M}\left(G \circ K_{q}\right) .
$$

\section{References}

[1] T. Bartnicki, B. Bresar, J. Grytczuk, M. Kovse, Z. Miechowicz, I. Peterin, Game chromatic number of Cartesian product graphs, Electron. J. Combin. 15, R72, 13 pp., 2008.

[2] J. Beck, Combinatorial Games: Tic-tac-toe theory, Cambridge University Press, 2008.

[3] E. R. Berlekamp, J. H. Conway, and R. K. Guy, Winning Ways for your Mathematical Plays. Vol. 1. A K Peters Ltd., Natick, MA, 2nd Ed., 2001.

[4] B. Bresar, S. Klavzar, D. F. Rall, Domination game and an imagination strategy, SIAM J. Discrete Math., 24, 979-991, 2010.

[5] B. Bresar, S. Klavzar, D. F. Rall, Domination game played on trees and spanning subgraphs, Discrete Math., 313 (8), 915-923, 2013.

[6] C. Bujt $\tilde{A}_{j \mathrm{~S}}$, Domination game on forests, 2014arXiv1404.1382B, 2014.

[7] G. Chartrand, L. Lesniak, P. Zhang. Graphs and Digraphs, 5th Ed., CRC Press, Boca Raton, 2011.

[8] T. Dinski, X. Zhu, Game chromatic number of graphs, Discrete Math. 196, 109115,1999

[9] P. Dorbec, G. Kosmrlj, G. Renault, The domination game played on unions of graphs, manuscript, 2013.

[10] L. Esperet, X. Zhu, Game colouring of the square of graphs, Discrete Math. 309, 4514-4521, 2009.

[11] U. Faigle, U. Kern, H. Kierstead, and W. T. Trotter. On the game chromatic number of some classes of graphs. Ars Combin., 35, 143-150, 1993. 
[12] A. Fraenkel, Combinatorial games: selected bibliography with a succinct gourmet introduction, Electron. J. Combin. DS2, 88 pp., 2009.

[13] M. Gardner, Mathematical games, Scientic American 244, 18-26, 1981.

[14] W. Goddard, A. Sinko, P. Slater and H. Xu, The graph distance game, AKCE Int. J. Graphs Comb., 8 (1), 85-96, 2011.

[15] D.J. Guan, X. Zhu, Game chromatic number of outerplanar graphs, J. Graph Theory, 30, 67-70, 1999

[16] J. Y. Kim, The incidence game chromatic number of paths and subgraphs of wheels, Discrete Appl. Math., 159 (8), 683-694, 2011.

[17] J. Nesetril, E. Sopena, On the oriented game chromatic number, Electron. J. Combin. 8 R14, 13pp., 2001.

[18] J.B. Phillips and P.J. Slater. Graph competition independence and enclaveless parameters. Congr. Numer., 154, 79-100, 2002.

[19] S.J. Seo and P. Slater, Competition parameters of a graph, AKCE Int. J. Graphs Comb., 4 (2), 183-190, 2007.

[20] E. Sidorowicz, The game chromatic number and the game colouring number of cactuses Information Processing Letters, 102 (4), 147-151, 2007

[21] M. Walsh. The competition chromatic numbers of a graph. Congr. Numer., 164,153-158, 2003.

[22] X. Zhu, Game coloring the Cartesian product of graphs, J. Graph Theory 59, 261-278, 2008. 